TRANSACTIONS OF THE

AMERICAN MATHEMATICAL SOCIETY

Volume 348, Number 8, August 1996

\title{
EXTENSIONS OF CODIMENSION ONE IMMERSIONS
}

\author{
CHRISTIAN PAPPAS
}

\begin{abstract}
We give a method for constructing all of the extensions of an immersion, and determine the CW structure and diffeomorphism type of each.
\end{abstract}

\section{INTRODUCTION}

The main results of this paper are a Morse-type theory for extensions, and a description of all of the inequivalent extensions of an immersion.

Let $f: M \rightarrow W$ be a transverse immersion of a closed orientable $m$-manifold $M$ into a connected orientable $(m+1)$-manifold $W$. Let $N$ be a compact $(m+1)$ manifold whose boundary is identified with $M$. An immersion $F: N \rightarrow W$ such that $\left.F\right|_{\partial N}=f$ is said to extend $f$ (in the direction of the inward pointing normal vectorfield on $F(\partial N)$ ). Two such extensions are considered equivalent if one can be obtained from the other by precomposition with a diffeomorphism of $N$. Milnor was the first to construct examples of inequivalent immersions of the disk in the plane which agreed on the boundary (see Figure 4.1 and [8]).

Extensions of an immersion $f: X^{p} \rightarrow Y^{q}$ of codimension greater than one may be treated by the Immersion theorem [2]. They are (up to regular homotopy) in one-to-one correspondence with (equivariant homotopy classes of) equivariant fiber map extensions of the induced map $D f: F X \rightarrow T_{p} Y$ of the frame bundle of $X$ to the bundle of $p$-frames of $Y$ (see $[2,8]$ ). In fact, much of Smale's work on immersions [10] concerns extending regular homotopies of immersed spheres to balls, which Hirsch [2] built an obstruction theory around.

Blank [8] determined the extensions of an immersed circle in the plane to a disk by employing the fundamental group of the image. This algebraic approach does not readily generalize (see $[7,6])$.

Theorem I, given in Section 3, describes the topology of an extension in terms of a handle structure on $N$. This handle structure is obtained from a Morse function $h: W \rightarrow \mathbf{R}$ such that the composition $h^{\prime}=h f$ is a Morse function on $M$. The handles of index $i$ come from the critical points of $h$ of index $i$, with multiplicities, and also from the "attaching" critical points of $h^{\prime}$ of index $i$, those critical points where the gradient flow of $h$ points into $N$. The multiplicities are determined by an integer invariant of an extension $F: N \rightarrow W$ called its grading, which is the minimum number of points of $F^{-1}(x)$ for $x \in W$. It follows that the various extensions of $f$, in the same direction and with the same grading, all satisfy the same Morse-type inequalities and have the same Euler characteristic. In addition,

Received by the editors January 1, 1995.

1991 Mathematics Subject Classification. Primary 57R42.

Key words and phrases. Extensions, immersions, codimension one, Morse theory.

(C)1996 American Mathematical Society 
the diffeomorphism type of an extension can be described, as for the usual Morse theory.

Theorem II, given in Section 4, describes all of the extensions of the immersion $f$, using the above setting. As one sweeps across the level sets of $h$, one attempts to build up an extension by confronting the topological and intersection set changes. The only obstructions are: the nonattaching critical points of $h^{\prime}=h f$ of index 1, and the 0-handles of the intersection locus (see Figures 4.2, 4.6a and 4.6b). Furthermore, there are local criteria for crossing these. There is (possibly) a choice involved with crossing 1-handles of $W$ and nonattaching critical points of $h^{\prime}$ of index 0, which accounts for inequivalent extensions (see Figures 4.1 and 4.3).

We conclude with a problem which concerns determining when extensions (with the same grading and direction) of a common immersion are diffeomorphic.

This paper is a generalization of the author's thesis [7].

\section{BASIC NOTIONS}

Let $M$ be a closed (compact, boundaryless) orientable smooth $m$-manifold, $W$ a connected orientable smooth $(m+1)$-manifold. Let $f: M \rightarrow W$ be a smooth transverse immersion. Recall that $f$ is an immersion if and only if the induced map of the fibers of the tangent bundles, $D f_{x}: T_{x} M \rightarrow T_{f(x)} W$, is one-to-one for all $x \in M$. By transverse we will mean that the $k$ tangent planes at a $k$-fold self-intersection point of $f(M)$ have $k$ linearly independent normal vectors in $W$. Such immersions are dense in the space of all immersions.

Fix a Riemannian metric on $W$. The immersion $f$ extends to an immersion of its normal bundle $\nu=M \times[-1,1]$, with the image a neighborhood of $f(M)$. A normal vectorfield is a nonvanishing section of the normal bundle $\nu$. The two unit normal vectorfields provide two directions that a normal vectorfield can point in, as well as inducing two orientations of $\nu$.

Let $w$ be the unit normal vectorfield such that the induced orientation of $\nu$ together with that of $D f(T M)$ induces the orientation of $T W$ at each point. The direction of $w$ will be called the orientation induced direction. Similarly, a normal vectorfield induces an orientation of $M$.

Let $N$ be an orientable $(m+1)$-manifold, whose boundary, $\partial N$, is identified with $M ; i: M \rightarrow N$ denoting inclusion. Let $u$ be a nonvanishing inward pointing normal vectorfield on $\partial N \subset N$. A smooth immersion $F: N \rightarrow W$, such that $F \circ i=f$ (so $\left.\left.F\right|_{\partial N}=f\right)$ is said to extend $f$ in the direction $D F(u)$. We will call $N$ the extending manifold of $F$.

Let $G_{i}: N_{i} \rightarrow W, i=1,2$, be extensions of $f$ in the same direction, as above. $G_{1}$ is equivalent to $G_{2}$ if and only if there is a diffeomorphism $g: N_{1} \rightarrow N_{2}$ such that diagram (a) commutes.

Inequivalent extensions occur quit generally. In fact

Proposition 2.1. If there is an immersion of a manifold $M$ into $W$ that extends, then there exists immersions of $M$ into $W$ which have exactly $k$ extensions for all integers $k \geq 0$.

The proof is postponed to the end of this section.

Evidently, a necessary condition for $f: M \rightarrow W$ to extend is that $f(M)$ is homologically trivial in $W, f_{*}[M]=0 \in H_{m}(W, \mathbf{Z})$, where $[M]$ denotes a generator of $H_{m}(M, \mathbf{Z})$. This will be assumed throughout. 


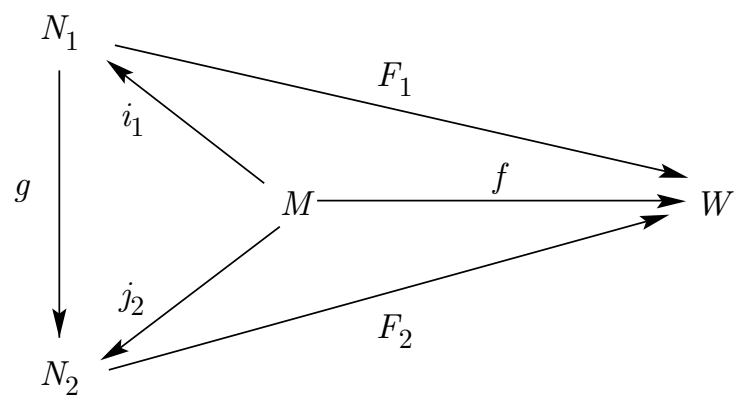

A component of the complement of the self-intersections of $f(M)$ in $f(M)$ will be called a wall; these are open subsets.

The image $f(M)$ cuts $W$ up into components. A wall is adjacent to a component if it is contained in the closure of the component in $W$. Each wall is adjacent to two distinct components; otherwise there would be a loop meeting $f(M)$ in exactly one point, contradicting the fact that $f(M)$ is homologically trivial in $W$.

Let $Y$ be the closure of a component of $W \backslash f(M)$. We leave to the reader to show that there is an immersion of a PL-manifold (more precisely, a smooth manifold with corners) $g: Y^{\prime} \rightarrow W$ such that $g\left(Y^{\prime}\right)=Y$ and any self-intersection of the immersion occurs in the boundary. The general case can be deduced from working an example, such as the immersion given in Figure 3.2.

The image of the PL-manifold is called a chamber. The boundary of a chamber refers to the image of the boundary of the PL-manifold. A compact chamber contained in the interior of $W$ is called a room. We will denote rooms by $A_{i}$, and their boundaries by $a_{i}$. On occasion, we will also refer to the PL-manifold as a chamber (or a room).

Two chambers (or their boundaries) with a wall in common are called neighbors; that common wall separates the chambers.

Let $\left.f\right|_{\mathrm{im}}: M \rightarrow f(M)$ denote the restriction of $f$ to its image, and $i: f(M) \rightarrow W$ denote inclusion. So $f=\left.i f\right|_{\mathrm{im}}$. Since $f_{*}[M]=0 \in H_{m}(W, \mathbf{Z})$ and $\left.f\right|_{\mathrm{im}^{*}}[M] \neq 0 \in$ $H_{m}(f(M), \mathbf{Z})$, we must have $\left.f\right|_{\mathrm{im}^{*}}[M] \in \operatorname{kernel}\left(i_{*}: H_{m}(f(M), \mathbf{Z}) \rightarrow H_{m}(W, \mathbf{Z})\right)$ which is generated by the boundaries of the rooms. So write $f_{*}[M]=\sum n_{i} a_{i}$. Now $f(M)$ contains exactly one copy of each wall, so if $a_{i}$ and $a_{j}$ are neighbors, then $\left|n_{i}-n_{j}\right|=1$.

If $W$ is not closed, then the $n_{i}$ are uniquely determined. If $W$ is closed, then the union of the rooms is $W$, so the sum of the $a_{i}$ is zero in $H_{m}(f(M), \mathbf{Z})$. Thus $f_{*}[M]$ has infinitely many different representations in $H_{m}(f(M), \mathbf{Z})$. Let $F: N \rightarrow W$ be an extension of $f$. Let $m_{i}$ be the number of components of $F^{-1}\left(A_{i}\right)$. So $F(N)$ is the union (over $i$ ) of $m_{i}$ copies of $A_{i}$, and $f_{*}[M]=\sum m_{i} a_{i} \in H_{m}(f(M), \mathbf{Z})$. The grading of $F, g(F)$, is the minimum (over $i$ ) of the $m_{i}$. If $W$ is not closed, then the grading must be zero.

An elementary example of grading is the immersion $f: S^{1} \rightarrow S^{2}$ occurring in Figure 2.1a, below. There is one extension of grading 0 , diffeomorphic to the torus minus a disk (indicated in Figure 2.1b). There are two inequivalent extensions of grading 1, both diffeomorphic to the disk (indicated in Figures 2.1c and 2.1d).

Lemma 2.2. There are only a finite number of extensions of a fixed grade. 


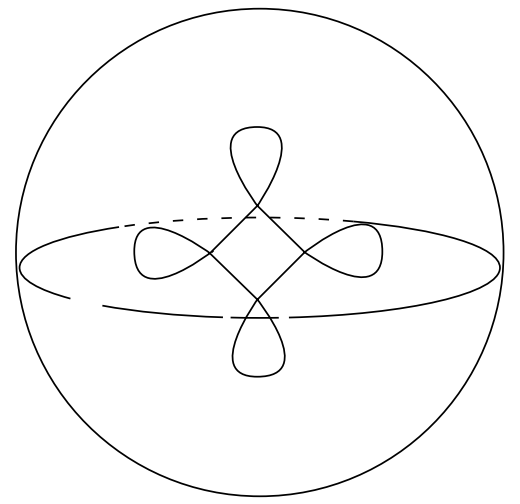

(a)

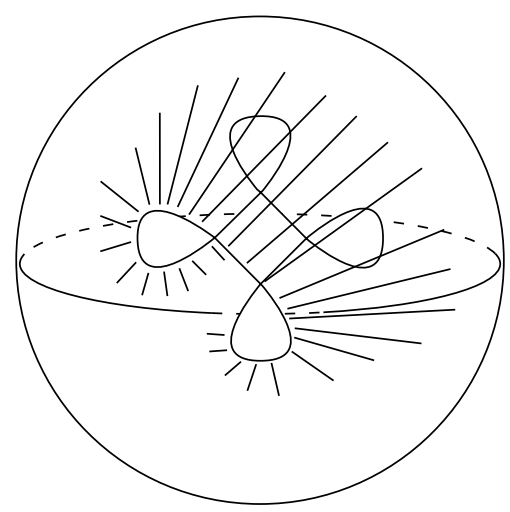

(c)

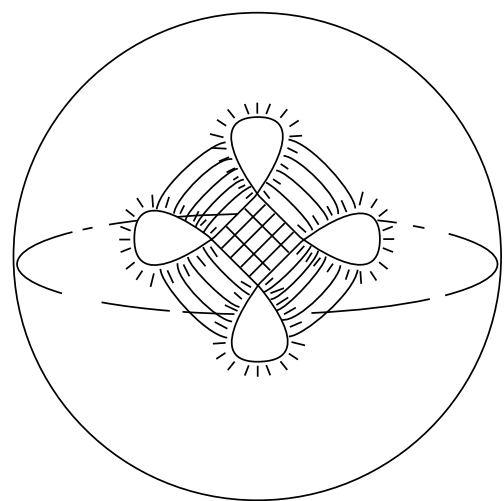

(b)

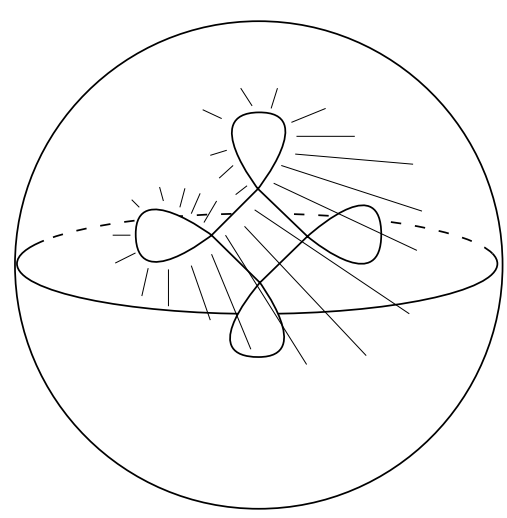

(d)

Figure 2.1. (a) $S^{1}$ in $S^{2}$. (b) Extension to $T^{2} \backslash$ disk. (c) An extension to a disk. (d) The other extension to a disk.

Proof. Such an extension is formed by gluing together a fixed number of copies of the rooms along their walls. The number of possible gluings, and thus extensions, is finite.

A proper path $g$ in $W$ is one whose endpoints are contained in the interior of chambers (which we will denote by the same letters as the chambers), and which meets $f(M)$ transversely at points in the walls of $f(M)$. Define the sum of the normal vectorfield $v$ along $g, \sum \operatorname{nvf}(g)$, to be the algebraic intersection of $f(M)$ with $g$, where $f(M)$ has the orientation induced from the normal vectorfield $v$.

Lemma 2.3. Let $g$ be a proper path, $A_{i}$ its initial point and $A_{t}$ its terminal point. Then $n_{t}-n_{i}=\sum \operatorname{nvf}(g)$. So if $g$ is a closed path, $\sum \operatorname{nvf}(g)=0$. Suppose $P$ is noncompact. Then $n_{t}=\sum \operatorname{nvf}(g)$, where $g$ is a path from an unbounded chamber to $A_{t}$.

Proof. By induction on the number of intersection points, we can reduce to just considering an arc $g$ between neighboring chambers $A_{i}$ and $A_{t}$, only meeting the 
wall $w$ separating them. It is now easy to check that $n_{t}-n_{i}=\sum \operatorname{nvf}(g)$, as each side is clearly 1 or -1 .

The remaining parts are immediate.

We end this section with the useful notion of matching.

Definition 2.4. A match for a path $g$ in $W$ (meeting $f(M)$ transversely) is a collection of disjoint matching pairs of the intersection points (of $g$ with $f(M)$ ), where a matching pair is a pair whose normal vectors point toward each other along $g$.

An extension $F: N \rightarrow W$ of an immersion $f$ induces a match of any path in $W$ as follows. The pre-image by $f$ of a path is a union of disjoint $\operatorname{arcs}$ in $N$. Consider those arcs whose boundary is contained in $\partial N=M$. The two endpoints of such an arc are naturally matched. So match the corresponding image points.

Lemma 2.5. Connected extensions are equivalent if and only if they induce the same match along any path.

Proof. Equivalent extensions just differ by pre-composition with a diffeomorphism which fixes the boundary $M$, so the induced matches agree.

Conversely, given extension $F_{i}: N_{i} \rightarrow W, i=1,2$, inducing the same match, we are to define the appropriate commuting map $g: N_{1} \rightarrow N_{2}$. Set $g$ to be the identity on the boundary. Fix a point $a \in \partial N_{1}$. Take $x \in N_{1}$ and an arc $j_{1}$ from $a$ to $x$ in $N_{1} . F_{2}^{-1} F_{1}\left(j_{1}\right)$ contains an arc $j_{2}$ such that one endpoint is $g(a)$. Set $g(x)$ to be the other endpoint of $j_{2}$.

Let $k_{1}$ denote another arc in $N_{1}$ from $a$ to $x . F_{2}$ induces the same match as $F_{1}$, so $F_{2}^{-1} F_{1}\left(k_{1}^{-1} j_{1}\right)$ contains an arc from $g(a)$ to itself. This arc contains $j_{2}$ and $k_{2}$, and so must be $k_{2}^{-1} j_{2}$. In particular, $j_{2}$ and $k_{2}$ have the same endpoints. Thus the map $g$ is well-defined. Furthermore, this shows that $F_{2}\left(j_{2}\right)=F_{1}\left(j_{1}\right)$.

One can define a map $h: N_{2} \rightarrow N_{1}$ in a similar manner as $g$, using arcs emanating from $g(a)$. Since $F_{1}\left(j_{1}\right)=F_{2}\left(j_{2}\right), j_{1} \subseteq F_{1}^{-1} F_{2}\left(j_{2}\right)$, so $h$ is the left inverse of $g(h g=$ identity). Similarly, $g$ is the left inverse of $h$. Thus $g$ is one-to-one and onto.

By construction, $g: N_{1} \rightarrow N_{2}$ is a diffeomorphism such that $F_{1}=F_{2} g$.

We will see at the end of Section 4 that just a finite number of paths suffice to pin down a match, when comparing extensions of a fixed grade.

We now give a proof of Proposition 2.1:

We first show that there exists an immersion of $S^{n}$ to $\mathbf{R}^{n+1}$ with exactly $k$ extensions, for $k \geq 0$.

Figure 2.2 visually lists immersions of the circle in the plane which admit exactly $k$ extensions, $k \geq 0$ (see [8]). A pattern develops with $k=1$.

Given an immersion $f: S^{n} \rightarrow \mathbf{R}^{n+1} \subset \mathbf{R}^{n+2}$ with exactly $k$ extensions, one may revolve it in $\mathbf{R}^{n+2}$ to obtain an immersion $F: S^{n} \times S^{1} \rightarrow \mathbf{R}^{n+2}$ with exactly $k$ extensions. One may perform "surgery" on the map $F$ to obtain an immersion $G: S^{n+1} \rightarrow \mathbf{R}^{n+2}$ with exactly $k$ extensions. Let $V$ be a disk neighborhood of a point $x \in S^{n}$. Essentially, one removes $f\left(V \times S^{1}\right)$ from $f\left(S^{n}\right)$ and glues in a copy of $S^{n-1} \times D^{2}$, and rounds corners.

Now consider an immersion $f: M^{m} \rightarrow W^{m+1}$ as before, which has $K>1$ extensions. We show how to regularly homotope $f$ to an immersion with $L$ extensions where $1 \leq L<K$. Since $f$ has more than one extension, there is a path $j$ which has at least two distinct induced matchings. Consider a wall $w$, meeting $j$ at a point $x$, 
Number of extensions

0

1

2

3

$\circ \circ \circ$
The immersion
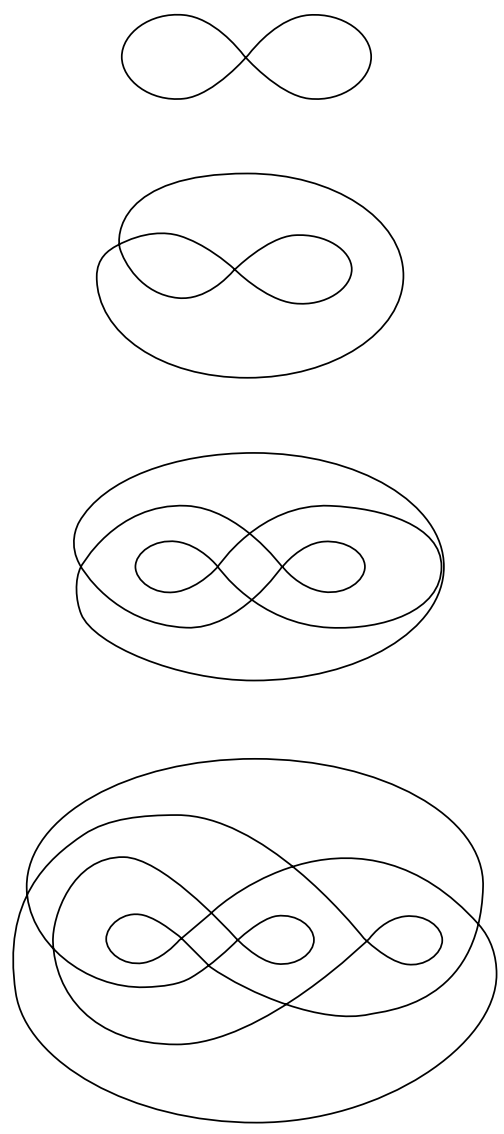

Figure 2.2. Circles in the plane with multiple extensions

which is matched to more than one wall along $j$. Suppose that $z$, meeting $j$ at $u$, is the closest wall matched to $w$ along $j$ (for some extension). Drag a neighborhood $U$ of $u$ along $j$ until $z$ is crossed. Now $w$ and $z$ can no longer match because the normals are now pointing away from each other along $j$. But $w$ can still match the walls that were further along $j$ than $z$. So the corresponding extensions persist.

By iterating the above, one arrives at an immersion $g: M^{m} \rightarrow W^{m+1}$ with exactly one extension. Let $g_{k}: S^{m} \rightarrow \mathbf{R}^{m}$ be an immersion with $k$ extensions. Let $V$ be an open ball in $S^{m}$. Remove an open ball from $f(M)$, and glue in a small copy of $g_{k}\left(S^{m} \backslash V\right)$ along their boundaries so as the normal vectorfields agree, and round corners. We have just obtained an immersion with $k$ extensions. 


\section{The TOPOLOGY OF EXTENSIONS}

Let $M$ be a connected closed orientable $m$-manifold, $W$ a connected orientable $(m+1)$-manifold. Let $f: M \rightarrow W$ be a transverse immersion and fix a normal vectorfield $v$. Let $h: W \rightarrow \mathbf{R}$ be a Morse function on $W$ such that the critical points of $h$ miss $f(M)$, and $h^{\prime}=h f$ is a Morse function on $M$. Such a function is obtained by perturbing a Morse function on $W$ in a neighborhood of $f(M)$, as Morse functions are dense in the space of functions.

All extensions in the direction $v$, with the same grading, contain the same number of copies of a given room $A$, called its multiplicity $m(A)$.

A critical point $c$ of $h$ such that $c$ is contained in a room $A$ with nonzero multiplicity will be called an ambient point. $c$ has multiplicity $m(c)=m(A)$.

Consider a critical point, $x$, of $h^{\prime}=h f$. Identify the tangent space of $\mathbf{R}$ at a point $y, T_{y} \mathbf{R}$, with $\mathbf{R}$ itself. $h$ induces a map, denoted $D h$, from the normal bundle of $f(M)$ to the tangent bundle of $\mathbf{R}, T \mathbf{R}$. If $D h_{x}(v(x)) \subset \mathbf{R}$ points in the positive direction, call $x$ an attaching point, else it is nonattaching. Figure 3.1a depicts an attaching point of index $q$. There is a handle consisting of the ascending and descending disks with the normal vectorfield drawn on each. Similarly, Figure 3.1b depicts a nonattaching point. One can equivalently define an attaching point $c$ as a critical point of $h^{\prime}$ such that $v$ points in the same direction as the gradient flow at $c$.

The index of an ambient point $c$ is the index of $c$ as a critical point of $h$; the index of an attaching point $c$ is the index of $c$ as a critical point of $h^{\prime}=h f$.

It would be instructive for the reader to examine Figures 3.2 and 3.3 to gain intuition for how the attaching points (indicated with arrows) contribute a handle to the extension.

Theorem I. Let $M$ be a connected closed orientable $m$-manifold, $W$ a connected orientable $(m+1)$-manifold. Let $f: M \rightarrow W$ be a transverse immersion, fix a normal vectorfield $v$, and fix the grading. Then every extension has a handle structure with $m(c)$ handles of index $i$ for each critical point $c$ of $h$ of index $i$, and one handle of index $i$ for each attaching point of $h^{\prime}=h f$ of index $i$.

If $M$ is disconnected, then the same statement holds as long as the extending manifold, $N$, is connected.

All of Morse theory carries over to this setting, so one can determine the diffeomorphism type of the extensions as well.

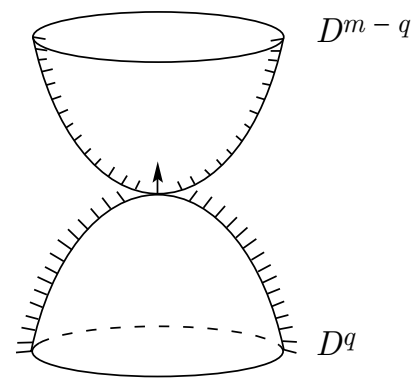

(a)

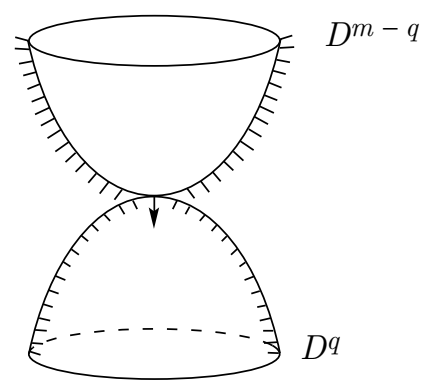

(b)

Figure 3.1. (a) Attaching point. (b) Nonattaching point. 


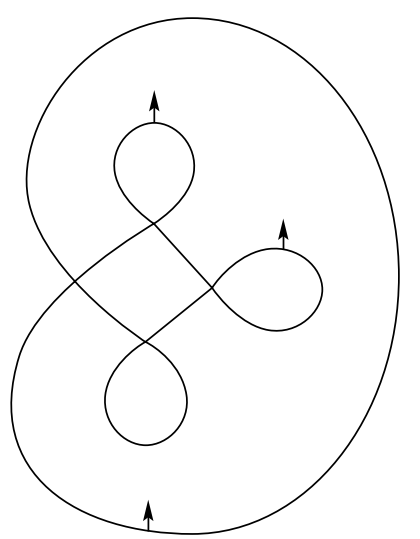

Figure 3.2. $S^{1}$ in $\mathbf{R}^{2}$.

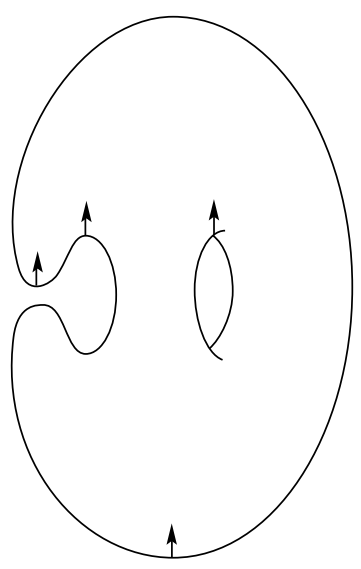

FiguRe 3.3. $T^{2}$ in $\mathbf{R}^{3}$.

Corollary 3.1. Extensions of the same grading satisfy the same Morse-type inequalities, and thus have the same Euler characteristic: Given any field $F$ as coefficient group, let $R_{q}(M)=$ qth Betti number of $M=\operatorname{rank}$ over $F$ of $h_{q}(M, F)$. Let $C_{q}=$ the number of ambient (with multiplicities) and attaching points of index $q$. Then

$$
R_{q}(M)-R_{q-1}(M)+\cdots \pm R_{0}(M) \leq C_{q}-C_{q-1}+\cdots \pm C_{0} .
$$

Corollary 3.2. Let $f: M^{1} \rightarrow F^{2}$ be an immersion, where $M$ is a closed 1-manifold (i.e. union of circles) and $F$ is a connected orientable surface. Then all connected extensions in the same direction with the same grading are diffeomorphic.

Proof. An orientable surface is determined by its Euler characteristic and the number of boundary circles.

Proof of Theorem I. Let $N$ be a compact connected orientable $(m+1)$-manifold whose boundary is identified with $M$. Let $F: N \rightarrow W$ be an immersion which extends $f$, so $\left.f\right|_{\partial N}=f: M \rightarrow W$. Let $h: W \rightarrow \mathbf{R}$ be a Morse function. Let $H=h F$. 
$F$ can be perturbed locally so that $H$ restricted to the interior of $N,\left.H\right|_{\operatorname{int}(N)}$, and to the boundary of $N,\left.H\right|_{\partial N}$, are Morse functions, and such that all critical points occur at different levels (as such functions are dense in the space of functions).

Each ambient point $c$ of index $i$ determines $m(c)$ interior critical points. Each interior critical point of index $i$ determines a handle of $N$ of index $i$.

Each boundary critical point $c$ of index $i$ determines a handle of $\partial N$ and thus of $N$ of index $i$. But if the boundary critical point is nonattaching (the normal vector at $c$ points to the descending disk), then the descending disk is filled in and hence cancelled. Thus only the attaching boundary critical points contribute to $N$.

The diffeomorphism type of $N$ can be determined by induction. Between consecutive ambient or attaching critical points, the extension is diffeomorphic to a product. Thus a handle can be viewed as being attached to the next lower proper level set via a retraction, which conforms to the immersion $f(M)$.

\section{EXTENDING AN IMMERSION}

Let $M$ be a closed orientable $m$-manifold, $W$ a connected orientable $(m+1)$ manifold. Let $f: M \rightarrow W$ be an immersion. We will give a procedure which yields all extensions, including ones which are disconnected.

Consider a Morse function $h$ on $W$ which has at most one critical point of index 0 and one of index $m+1$. Set $P(t)=h^{-1}(t)$, the level set at $t$.

Let $Y^{(k)}=\left\{y \in W: y=f\left(x_{1}\right)=\cdots=f\left(x_{k}\right) ; x_{1}, \ldots, x_{k}\right.$ distinct elements of $M\}, k \geq 2$. $Y^{\prime \prime}$ is the intersection locus of $f(M) ; Y^{(k+1)} \subseteq Y^{(k)}$. There exists an immersion $f_{k}: X^{(k)} \rightarrow Y^{(k)}$ of an $(m+1-k)$-manifold $X^{(k)}$ onto $Y^{(k)}$. Let $h^{(k)}=h f_{k}$. As a notational convenience, set $X^{0}=Y^{0}=W, f_{0}=$ identity, $h^{0}=h$, $X^{\prime}=M, Y^{\prime}=f(M), f_{1}=f$ and $h^{\prime}=h f$.

Without affecting whether $f$ extends or not, $f$ can be perturbed locally so that $h^{(k)}$ is a Morse function on $X^{(k)}$ whose critical points miss $f_{k}^{-1}\left(Y^{(k+1)}\right), k \geq 0$, and these critical points occur at different levels (as such functions are dense in the space of functions).

We construct extensions of $f$ by building up the extending manifolds as we sweep across the level sets of $h$ simultaneously producing the immersion $F$. The only potential obstructions are the critical points of $h^{(k)}, k \geq 0$.

A level extension of $P(s)$ (i.e. at $s$ ) is an extension of $f(M) \cap P(s)$ in $P(s)$ (i.e. of the restriction $\left.f \mid f^{-1}(P(s)) \rightarrow P(s)\right)$. To piece together level extensions, they need to vary smoothly; meaning that there are immersions of extensions in a neighborhood of each level extension, called a slab of extensions. An extension up to $t$ consists of a smooth variation of level extensions up to $P(t)$.

Say $c$ is a critical point of $h^{(k)}$, let $t=h^{(k)}(c)$. The limit of the level extensions of $P(r)$, for $r<t$, will be called the candidate of $c$.

Let $V$ be a small open neighborhood of $f_{k}(c)$ in $W$. An extension restricted to $P(t+\varepsilon) \backslash V$ would exactly agree with the extension restricted to $P(t-\varepsilon) \backslash V$, as any changes occur in $V$. More precisely, we may assume that outside of $V$ $f(M) \cap h^{-1}([t-\varepsilon, t+\varepsilon])$, is "foliated" by the flow lines of the gradient vectorfield of $h$, by gently perturbing $f(M)$. Then one can use the gradient flow to regularly homotope the extension restricted to $P(t-\varepsilon) \backslash V$ to $P(t+\varepsilon) \backslash V$.

Thus one may focus on a neighborhood of a critical point to determine whether one can construct a slab of extensions about it. Doing so will be called crossing the critical point. Constructing a level extension from a previous one will be called 


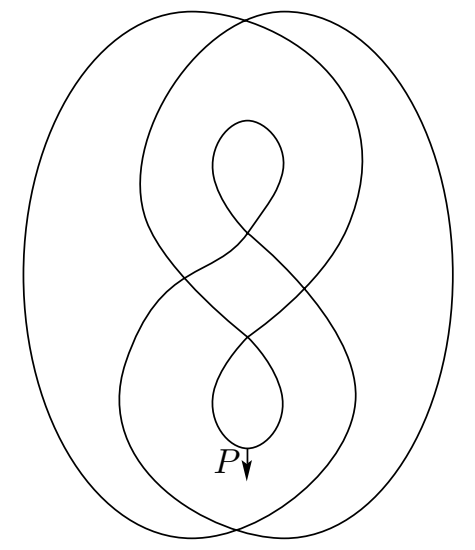

Figure 4.1. $S^{1}$ in $\mathbf{R}^{2}$. Two extensions

transporting the extension. We will often describe how to cross a critical point by just explaining how the image of the level extensions change about it.

Theorem II. Let $M$ be a closed orientable m-manifold, and $W$ a connected orientable $(m+1)$-manifold. Let $f: M \rightarrow W$ be a transverse immersion, $v$ a normal vectorfield, and fix the grading. Assume that $f(M)$ is homologically trivial in $H_{m}(W)$. Let $h$ be a Morse function on $W$ as above. As one sweeps across the level sets, one only needs to consider the following in constructing extensions (the remaining critical points can always be crossed in a unique way).

The obstructions are:

1. Nonattaching critical points of $h^{\prime}$ of index 1 . One can be crossed (in a unique way) if and only if points of the descending 1-disk are matched in a neighborhood of it.

2. 0-handles of intersection components. One can be crossed (in a unique way) if and only if the sheets of $f(M)$ containing it are not matched in a neighborhood of it.

The following critical points can always be crossed, but there may be more than one way to do so:

3. Nonattaching critical points of $h^{\prime}$ of index 0 . The number of ways to cross such a point $c$ equals the multiplicity of the room that $v$ at $c$ points to.

4. Critical points of $h$ of index 1 . There are $m(c)$ ! ways to cross such a point $c$, corresponding to pairing off sheets at the ends of the descending 1-disk.

The choices made in crossing nonattaching 0-handles and 1-handles of $W$ are exactly what allows for inequivalent extensions (though the possibilities of the latter may lead to redundancies).

The end of this section reformulates Theorem II in a slightly different context.

The points listed in Theorem II are depicted in Figures 4.1, 4.2, 4.3, 4.6a and $4.6 \mathrm{~b}$ (discussed later). Figure 4.1 captures the essence of crossing a nonattaching 0 -handle (denoted $P$ ). There are two extensions of this circle immersed in $\mathbf{R}^{2}$. Figure 4.2 illustrates crossing a nonattaching 1-handle (denoted $Q$ ) with an immersed 2 -sphere in $\mathbf{R}^{3}$. There is one extension. 


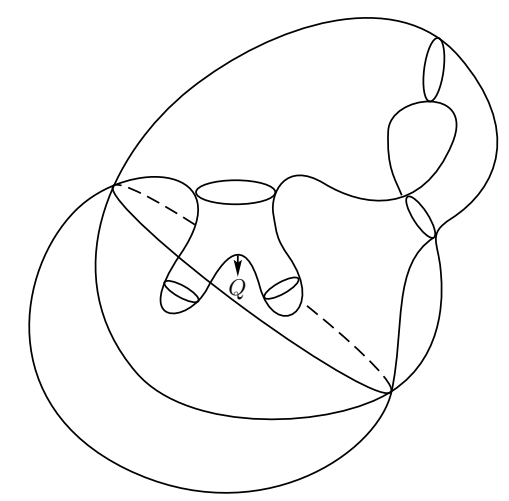

FiguRE 4.2. $S^{2}$ in $\mathbf{R}^{3}$. One extension
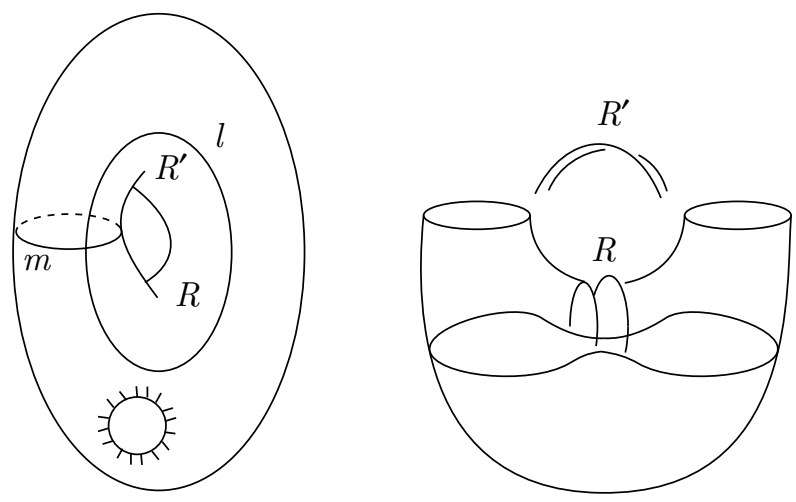

FiguRE 4.3. $S^{1}$ in $T^{2}$

Figure 4.3 consists of a circle embedded in the 2-torus, with a normal vectorfield pointing away from the disk that it bounds, to demonstrate crossing ambient 1handles. The grading 0 extension consists of the torus minus the disk. There are exactly four inequivalent extensions of grading 1 . One is disconnected, being the union of the torus with the grading 0 extension. The connected extensions can be obtained from the following double covers of the torus (by removing a disk from the pre-image). Let $l$ and $m$ denote the standard longitude and meridian of $T^{2}$, as indicated in the figure. The three convergings are the ones induced by the following maps of fundamental groups, given by: $l \rightarrow 2 l, m \rightarrow m ; l \rightarrow l, m \rightarrow 2 m ; l \rightarrow l+m$, $m \rightarrow-1+m$. Higher grading extensions are constructed similarly.

Proof of Theorem II. Let us consider constructing an extension of a fixed grade, $g$. It will be denoted $F: N \rightarrow W$.

Concerning $W$, we now show that all ambient critical points can be crossed, but that the 1-handles may be crossed in more than one way.

Lemma 4.1. Let $c$ be a critical point of $h$. Then one can cross $c$. If the index of $c$ is 0 or greater than 1 , then there is a unique way to cross $c$. 
Proof. $h$ has exactly one critical point $e$ of index 0 , which is the first point encountered as we sweep across $W$. Crossing $e$ simply corresponds to introducing $m(e)$ 0 -handles of $F(N)$. Clearly, there is a unique way to do this.

Let $c$ be a critical point of index $q>1$; let $t=h(c)$. Say $c$ is contained in chamber $A$. We assume we have an extension up to $t-\varepsilon$. One can show that the extension meets a chamber $B m(B)$ times by using an inductive argument as in the proof of Lemma 2.1.

A neighborhood of $f(c)$ in $f(M)$ consists of a descending $q$-disk and an ascending $(m+1-q)$-disk. $P(t-\varepsilon)$ meets the descending $q$-disk, which is thickened up to $D^{q} \times D^{m+1-q}$, in $S^{q-1} \times D^{m+1-q} . P(t+\varepsilon)$ meets the ascending $(m+1-q)$-disk, which is thickened up to $D^{q} \times D^{m+1-q}$, in $D^{q} \times S^{m-q}$. So one can produce a level extension at $t+\varepsilon$ via $m(A)$ surgeries on the level extension at $t-\varepsilon$. Each surgery corresponds exactly to the change in the level sets of $W$. So $m(A)$ copies of $D^{q} \times D^{m+1-q}$ are adjoined as one crosses $c$.

If $q>1$, then $S^{q-1}$ and thus $S^{q-1} \times D^{m+1-q}$ is connected. So there is a unique way to perform the surgery.

Lemma 4.2. Let $c$ be a critical point of $h$ of index 1 .

1. The algebraic intersection of the descending 1-disk with a level extension is zero.

2. There are $m(c)$ ! ways that c can be crossed, corresponding to pairing off the sheets at the ends of the descending 1-disk.

Proof. The descending 1-disk, $u$, belongs to the same chamber as $c$, which we call $A ; m(c)=m(A)$. So each endpoint of $u$ meets $m(A)$ sheets; thus the algebraic intersection is zero.

The 1-disk $u$ joins up sheets of the level extension. So the number of ways that $c$ can be crossed equals the number of ways that the sheets at opposite endpoints of $u$ can be paired off. There are $m(A)$ ! ways of achieving this.

The level extension at $t+\varepsilon$ is obtained from the level extension at $t-\varepsilon$ by surgery, effected by adding in the boundary of $D^{1} \times D^{m} m(A)$ times.

As regards crossing critical points of $f(M)$, we now show that only the nonattaching ones of index 1 are an obstacle for extensions, and the nonattaching ones of index 0 may possibly be crossed in more than one way.

Lemma 4.3. Let $c$ be an attaching critical point of $h^{\prime}=h f$. Then $c$ can be crossed in a unique way.

Proof. Say the index of $c$ is $q$. A neighborhood $V$ of $f(c)$ in $f(M)$ consists of a descending $q$-disk and an ascending $(m-q)$-disk. The normal vector at $f(c)$ points to the $(m-q)$-disk. $P(t+\varepsilon)$ meets the ascending $(m-q)$-disk, which is thickened up to $D^{q} \times D^{m-q}$ in $D^{q} \times S^{m-q-1}$. $P(t-\varepsilon)$ meets the descending $q$-disk, which is thickened up to $D^{q} \times D^{m-q}$, in $S^{q-1} \times D^{m-q}$. See Figure 4.4a.

A level extension at $t+\varepsilon$ can be constructed by simply attaching a copy of $D^{q} \times D^{m-q}$ (a thickened $q$-disk) to the level extension at $t-\varepsilon$. There is a unique way to do this since it is attached to the boundary.

This accounts for the surgery change from $f^{-1} P(t-\varepsilon)$ to $f^{-1} P(t+\varepsilon)$ (in $M$ ), as the boundary of $D^{q} \times D^{m-q}$ is $S^{q-1} \times D^{m-q}$ union $D^{q} \times S^{m-q-1}$. By keeping track of orientations, one sees that to go from $f^{-1} P(t-\varepsilon)$ to $f^{-1} P(t+\varepsilon)$ (in $M$ ) one cuts out $S^{q-1} \times D^{m-q}$ and pastes in $D^{q} \times S^{m-q-1}$. See Figure 4.4b. 


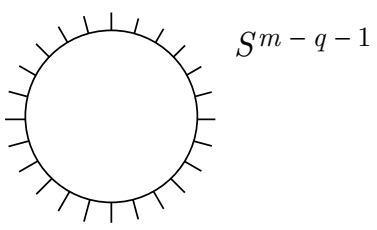

Level slices
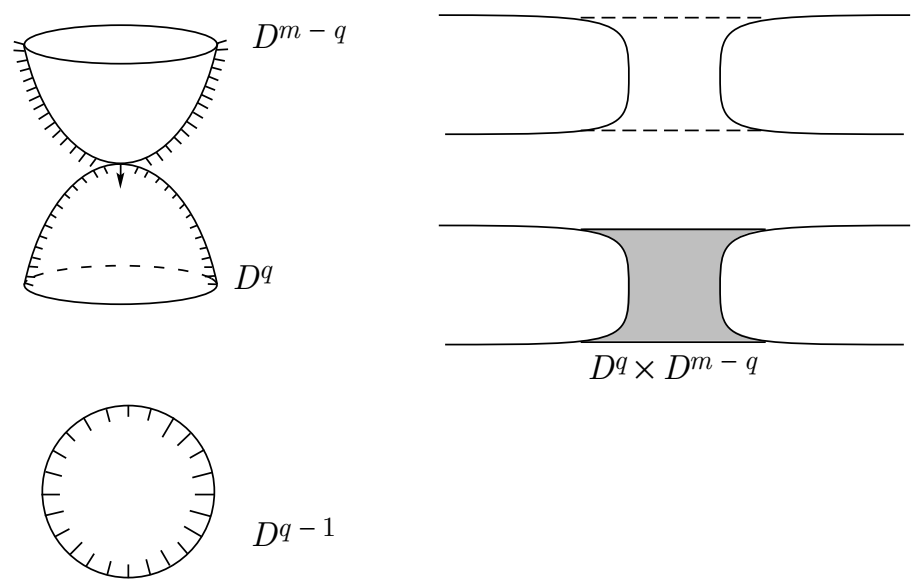

FiguRE 4.4. Crossing a nonattaching point

Lemma 4.4. Let $c$ be a nonattaching critical point of $h^{\prime}=h f$ of index $q>1$. Then one can cross $c$ in a unique way.

Proof. A neighborhood $V$ of $f(c)$ in $f(M)$ consists of a descending $q$-disk and an ascending $(m-q)$-disk, with the normal vectorfield pointing to the $q$-disk. Crossing a nonattaching point is the reverse of crossing an attaching one. So if there is an extension, then a level extension at $t+\varepsilon$ can be constructed by removing a copy of $D^{q} \times D^{m-q}$ from the level extension at $t-\varepsilon$. For $q>1$, we show that there is such a $D^{q} \times D^{m-q}$ to remove.

$P(t-\varepsilon)$ meets the descending $q$-disk, which is thickened up to $D^{q} \times D^{m-q}$, in $S^{q-1} \times D^{m-q}$. There is a $q$-disk $R$ in $P(t-\varepsilon)$ meeting the descending $q$-disk in a $(q-1)$-sphere $S$, which is connected.

We claim that the extension restricted to $R$ fills in the sphere; thus these points of the $(q-1)$-sphere must match themselves. Otherwise, suppose that they did not.

Let $U$ be a chart neighborhood of $c$ in $P(t-\varepsilon)$. We assume that $S$ is a round sphere contained in a $q$-dimensional hyperplane $P$ of $U$. Foliate $P$ by parallel lines, perpendicular to a $(q-1)$-dimensional hyperplane $Q$ of $P$, passing through the center of $S$. The lines passing through the points of the $(q-2)$-sphere $E=Q \cap S$ are tangent to $S$. $S \backslash E$ consists of two hemispheres $H_{1}$ and $H_{2}$. Any other line meets $S$ in zero or two points.

Let $g$ be an arc meeting $H_{1}$ and $H_{2}$ perpendicularly at points $a$ and $b$, respectively, and say that $g$ is contained in a small neighborhood of a leaf of the foliation. As $H_{1}$ and $H_{2}$ are not matched, we may prolong $g$ to an $\operatorname{arc} j$ in $P$ such that $a$ and $b$ are matched to points on $j$ which lie on opposite sides of $g$. The prolongations 


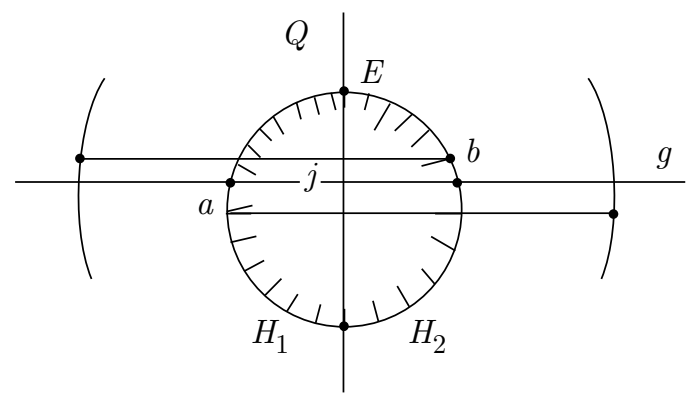

Figure 4.5. Small spheres extend to balls

may be chosen in a continuous fashion on $S \backslash E$, as $S$ is small. No extension could induce this matching, as it would be singular along $E$. See Figure 4.5.

So a level extension in $P(t-\varepsilon)$ must fill in the sphere $S$. Thus $F(M) \cap P(t-\varepsilon) \cap V$, identified with $S^{q-1} \times D^{m-q}$, extends to $D^{q} \times D^{m-q}$. This is what is removed.

If $q>1$, then $S^{q-1}$ and thus $S^{q-1} \times D^{m+1-q}$ is connected. So there is a unique way to perform the surgery.

Lemma 4.5. Let $c$ be a nonattaching critical point of $h^{\prime}$ of index 0 . Then one can cross $c$ in $m(A)$ ways where $A$ is the chamber that $v$ at $c$ points to.

Proof. Let $t=h(c)$. Consider a neighborhood of $f(c)$ in $f(M)$. Fix a level extension of $P(t-\varepsilon) . P(t+\varepsilon)$ meets the ascending $m$-disk in an $(m-1)$-sphere, $S$. So $f(M) \cap P(t+\varepsilon)$ is topologically and combinatorially the same as $f(M) \cap P(t-\varepsilon)$, except that the sphere $S$ is added in. The normal at $c$ points away from the ascending $m$-disk.

A level extension at $t+\varepsilon$ can be obtained from the level extension at $t-\varepsilon$ by removing an open $m$-ball (which is the interior of $S$ ). An $m$-ball can be removed from each sheet of the candidate of $c$ that meets $c$, which there are $m(A)$ of, all giving rise to inequivalent extensions up to $t+\varepsilon$. Note that $m(A)>0$ since $f_{*}[M]=0 \in H_{m}(W, Z)$.

Lemma 4.6. Let $c$ be a nonattaching critical point of $h^{\prime}$ of index 1 . Then $c$ can be crossed (in a unique way) if and only if points of the descending 1-disk are matched in a small neighborhood of it.

Proof. Let $t=h(c)$. Consider a neighborhood of $f(c)$ in $f(M)$. $P(t-\varepsilon)$ meets the descending 1-disk in two points, say $a$ and $b$.

Suppose that we have an extension of $f(M) \cap P(t-\varepsilon)$ which does not match $a$ and $b$; so locally, $a$ and $b$ lie in different sheets of the extension. Consider the limit of this level extension to $c$; as well as the limit of the two sheets, which are distinct. By continuity, $c$ belongs to both of these distinct sheets - a contradiction.

Now suppose that we have an extension of $f(M) \cap P(t-\varepsilon)$ which matches $a$ and $b$ in a small neighborhood of $c$. A level extension of $P(t+\varepsilon)$ can be obtained from the extension of $P(t-\varepsilon)$ by removing $D^{1} \times D^{m-1}$, which is a thickening of an arc from $a$ to $b$.

We now show that the only obstacles involved with intersections lie in crossing their 0 -handles. The following is a useful condition which we will employ. 


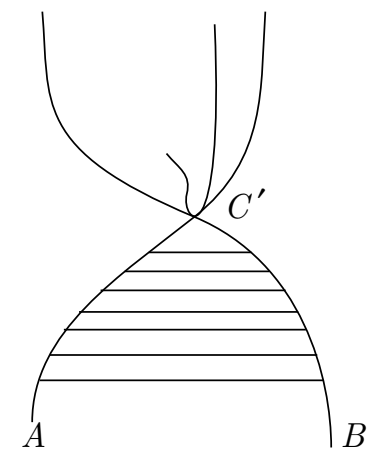

FiguRE 4.6a. Obstruction to crossing a 0-handle of the intersection locus.

Lemma 4.7. Let $c$ be a critical point of $h^{(k)}, k \geq 2$. $c$ can be crossed (in a unique way) if and only if the candidate of $c$ is an immersion of a manifold.

Proof. Let $t=h^{(k)}(c)$. There are no critical values of $h$ or $h^{\prime}$ in a neighborhood of $t$. So if there was a slab of level extensions about $c$, the domain would be a product manifold. Thus the candidate is an immersion of a manifold.

Conversely, if the candidate is an immersed manifold, then one could just regular homotope it across $c$. Explicitly: a level extension of $f(M) \cap P(t+\varepsilon)$ in $P(t+\varepsilon)$ can be obtained from a level extension of $f(M) \cap P(t-\varepsilon)$ in $P(t-\varepsilon), F_{t-\varepsilon}: L \rightarrow$ $P(t-\varepsilon)$, by a regular homotopy $j: L \times[-\varepsilon, \varepsilon] \rightarrow W$ such that $j(x, s) \in P(s)$ and $j(\partial L, s)=f(M) \cap P(s)$, for all $s \in[-\varepsilon, \varepsilon]$. The transport is unique since the domain of a regular homotopy is a product manifold.

Any singularity of the candidate must occur at the critical point. As outside a neighborhood of the critical point the candidate is an immersed manifold, obtained from a previous level by a regular homotopy.

Lemma 4.8. Let c be a critical point of $h^{\prime \prime}$ of index 0 ( $a$ 0-handle of the intersection locus). Then one can cross $c$ (in a unique way) if and only if the sheets of $f(M)$ meeting at $c$ are not matched in a small neighborhood of it.

Proof. Let $V$ be a small neighborhood of $c^{\prime}=f_{2}(c)$ in $W$. Let $t=h^{\prime \prime}(c)$. Choose $\varepsilon$ so small such that $P(t-\varepsilon)$ meets $V$. Let $A$ and $B$ be sheets of $f(M)$, contained in $V$, such that $c^{\prime} \in A \cap B$. Suppose we have an extension up to $t-\varepsilon$ which induces a match of $A \cap P(t-\varepsilon)$ and $B \cap P(t-\varepsilon)$ in $V$. Then the candidate of $c$ has a singularity (at $\left.c^{\prime}\right)$ and therefore we cannot cross $c$. See Figure 4.6a.

Now suppose that $A$ and $B$ are not matched. Then $A$ must match another sheet, $A^{\prime}$. The matching between points of $A$ and $A^{\prime}$ can be continued past the critical point $x$ (see Figure 4.6b). A similar situation holds for the sheet $B$. Thus a singularity will not develop at $c$. By Lemma 4.7 and the comment following it, $c$ can be crossed.

Lemma 4.9. Let $c$ be a critical point of $h^{\prime \prime}$ of index $q>0$. Then one can cross $c$ (in a unique way). 


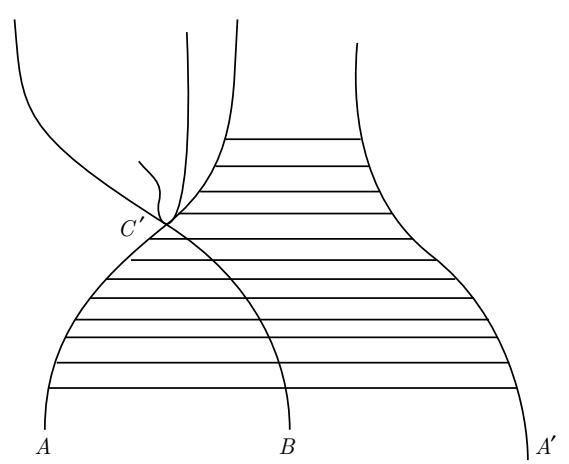

Figure 4.6b. Crossing a 0-handle of the intersection locus.

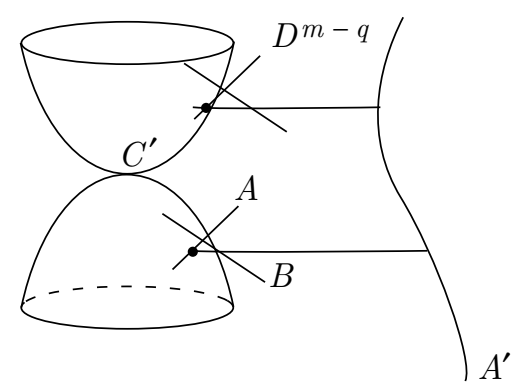

FIGURE 4.7. Crossing critical points of the intersection locus.

Proof. Let $V$ be a small neighborhood chart of $c^{\prime}=f_{2}(c)$ in $W$. Let $Z=V \cap Y^{\prime \prime}$. Let $t=h^{\prime \prime}(c)$. Choose $\varepsilon$ so small such that $P(t-\varepsilon)$ meets $V$. Let $A$ and $B$ be sheets of $f(M)$, contained in $V$, so $c^{\prime} \in Z=A \cap B$.

We describe now the local structure of $c^{\prime} \in A \cap B$ to enhance our intuition. Identify $V$ with $\mathbf{R}^{m+1}=\mathbf{R}^{m} \times \mathbf{R}^{1}$ where the first factor is identified with $P(t) \cap V$, and has local coordinates $\left\{x_{1}, \ldots, x_{m}\right\}$; and the second is spanned by the gradient flow at $c^{\prime}$, and has local coordinate $\left\{x_{m+1}\right\} . c^{\prime}$ occurs at the origin.

Furthermore, we assume that the intersection $Z=A \cap B$ is contained in the plane $Q$ defined by $x_{m}=0$. Now $Z=A \cap B$ is a $q$-handle, and so resembles the graph of $\left\{x_{m+1}=-x_{1}^{2}-\cdots-x_{q}^{2}+x_{q+1}^{2}+\cdots+x_{m-1}^{2}, x_{m}=0\right\}$.

The sheets $A$ and $B$ meet at $Z$. So $A(B)$ can be viewed as $Z \times[-1,1]$ but with the points near $Z \times\{1\}$ perturbed in the $+w(-w)$ direction, and the points near $Z \times\{-1\}$ perturbed in the $-w(+w)$ direction. So we can take $A$ to be the graph of $x_{m+1}=-x_{1}^{2}-\cdots-x_{q}^{2}+x_{q+1}^{2}+\cdots+x_{m-1}^{2}+x_{m}$, and $B$ the graph of $x_{m+1}=-x_{1}^{2}-\cdots-x_{q}^{2}+x_{q+1}^{2}+\cdots+x_{m-1}^{2}-x_{m}$. They intersect at the $q$-handle $Z$ in the plane $x_{m}=0$.

$A$ and $B$ cannot be matched. Otherwise the extension would have a singularity at points in $Z$. Thus $A \cap P(t-\varepsilon)$ is matched to $A^{\prime} \cap P(t-\varepsilon)$ for some sheet $A^{\prime}$ which does not meet $A$. The matching between points of $A$ and $A^{\prime}$ can be continued past the critical point $x$; and similarly for sheet $B$ (see Figure 4.7). This implies that a singularity does not occur. But we will finish off the proof another way. 
In our chart $V$, we may view $A$ and $B$ as graphs over the plane $Q$, which is perpendicular to $P(t)$. For instance, $A$ is defined by $x_{m}=x_{1}^{2}+\cdots+x_{q}^{2}-x_{q+1}^{2}-$ $\cdots-x_{m-1}^{2}+x_{m+1}$. Furthermore, we may assume that $Q$ is perpendicular to $P(s)$, for $s \in[t-\varepsilon, t+\varepsilon]$. One may regularly homotope a level extension of $P(t-\varepsilon)$ along the gradient flow to $P(t+\varepsilon)$ and then perturb it in $V$ in the direction perpendicular to $Q$ to obtain a level extension of $P(t+\varepsilon)$. There is no obstruction to doing this as $A$ and $B$ are nowhere matched in $V$. Thus one may cross $c$.

Lemma 4.10. Critical points of $h^{(k)}, k \geq 3$, can be crossed (in a unique way).

Proof. One can argue as in the previous lemma. Let $V$ be a neighborhood chart of $c^{\prime}=f_{k}(c)$ in $W$. Say the index of $c$ is $q$. Let $Z=V \cap Y^{k}$, so $c^{\prime} \in Z$. Let $t=h^{(k)}(c)$. Choose $\varepsilon$ so small such that $P(t-\varepsilon)$ meets $V$. Let $A_{1}, \ldots, A_{k}$ be sheets of $f(M)$, contained in $V$, such that $Z=A_{1} \cap \cdots \cap A_{k}$.

We may assume that the sheets $A_{1}, \ldots, A_{k}$ have the following form, by noting how one perturbs $k$ copies of $Z \times[-1,1]^{k-1}$ to produce $k$ sheets intersecting at $Z$. We take $A_{1}$ to be given by the graph of $x_{m+1}=-x_{1}^{2}-\cdots-x_{q}^{2}+x_{q+1}^{2}+$ $\cdots+x_{m+1-k}^{2}+x_{m+2-k}+\cdots+x_{m} ; A_{j}$ given by the graph of $x_{m+1}=-x_{1}^{2}-\cdots-$ $x_{q}^{2}+x_{q+1}^{2}+\cdots+x_{m+1-k}^{2}+x_{m+2-k}+\cdots+x_{m}-x_{m-k+j}$, for $j=2, \ldots, k-1$; and $A_{k}$ given by the graph of $x_{m+1}=-x_{1}^{2}-\cdots-x_{q}^{2}+x_{q+1}^{2}+\cdots+x_{m+1-k}^{2}+$ $x_{m+2-k}+\cdots+x_{m-1}-x_{m}$. These sheets intersect at $Z$ given by the graph of $\left\{x_{m+1}=-x_{1}^{2}-\cdots-x_{q}^{2}+x_{q+1}^{2}+\cdots+x_{m+1-k}^{2}, x_{m+2-k}=\cdots=x_{m}=0\right\}$.

As before, $A_{1}, \ldots, A_{k}$ can be viewed as graphs over the coordinate plane $Q$, given by $x_{m}=0$, which is perpendicular to $P(t-\varepsilon)$. One may regularly homotope a level extension of $P(t-\varepsilon)$ to $P(t+\varepsilon)$ and then perturb it in $V$ in the direction perpendicular to $Q$ to obtain a level extension of $P(t+\varepsilon)$. There is no obstruction to doing this as no pair of the sheets $A_{1}, \ldots, A_{k}$ can be matched to each other, since then a singularity would develop in $Z$.

Thus no critical point of $h^{(k)}, k \geq 3$, is an obstruction to extending.

To produce an extension, sweep across $W$ and check that each critical point can be crossed. The above lemmas describe how to build the extension. There may be a choice in crossing the 1-handles of $W$ and the nonattaching 0-handles of $f(M)$ which lead to inequivalent extensions. Note that there may be redundancies in the (finite) list of extensions provided by Theorem II, arising from crossing the 1-handles of $W$. Constructing grading two extensions of the example given in Figure 4.3 provides an instance of this.

We indicate how the above yields all of the extensions of $f$ of a fixed grade $g$. Suppose that $F^{\prime}: N^{\prime} \rightarrow W$ is an extension of $f$ of grading $g$. One can explicitly construct a diffeomorphism of $F^{\prime-1} h^{-1}((-\infty, t])$ with exactly one of the above extensions up to $t$, for all $t$, by mapping the level sets in a smooth fashion. Let $e$ denote the absolute minimum of the extension. If $e$ is an ambient point, then we start with $m(e)$ sheets in a neighborhood of $e$ of the extension; if $e$ is an attaching point, then we start with one sheet. The maps are prescribed by the lemmas.

This concludes the proof of Theorem II.

We reformulate Theorem II to provide an alternative method for producing extensions.

The level system consists of the level sets containing the nonattaching points of index 0 and 1, the 0-handles of the intersection locus and the 1-handles of $W$. For each such level set, consider all possible slabs of extensions about it. Now fix 


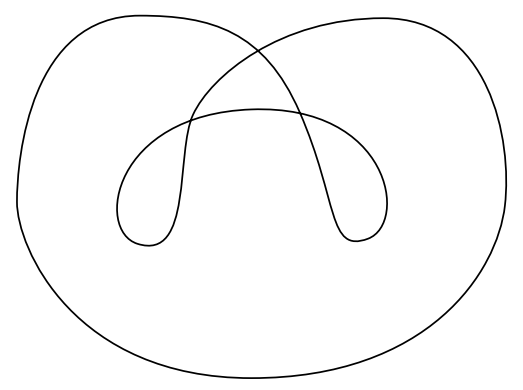

FiguRE 4.8. Incompatible intersections

two successive level sets $P$ and $Q$, and two slabs of extensions about them, $P^{\prime}$ and $Q^{\prime}$. We know that the slab extensions $P^{\prime}$ can be transported to the level set $Q$, by Theorem II. If this level extension agrees with the slab extension $Q^{\prime}$, then we will say that the slab extensions $P^{\prime}$ and $Q^{\prime}$ are compatible.

Constructing an extension thus reduces to producing slabs of extensions about each level set of the level system which are compatible. The following gives a useful method to check for compatibility.

An extension is determined by its induced match. In fact, just knowing matches along a finite number of select paths pins down an extension of a fixed grade (provided we know that it exists), since the choices for an extension occur at the nonattaching 0 -handles of $M$ and the 1-handles of $W$. Thus to show compatibility, one just needs to transport the matches in an appropriate set of select paths of $P$ and check that this pins down the match, and hence extension in $Q$. In general, one only needs to transport matches in $n(Q)-1$ select paths, where $n(Q)=$ the number of level extensions of $Q$. Presently, there is no general scheme for transporting these paths, though it yields to many specific cases. For example, if the level extension at $Q$ is unique, then it must be equivalent to the transported extension; so the level extensions of $P$ and $Q$ are automatically compatible.

Figure 4.8 is an example of a circle immersed in the plane with incompatible intersections.

\section{Conclusion}

Immersions of the 2-sphere in Euclidean 3 -space provide a natural setting for a further study of extensions.

Let $N$ be a closed orientable 3-manifold, $B$ a small open ball in $N . N \backslash B$ can be immersed into $\mathbf{R}^{3}$ (consult [11] or [2]). Denote the immersion by $F: N \backslash B \rightarrow \mathbf{R}^{3}$. The boundary of $N \backslash B$ is a 2-sphere, $S^{2}$. So $F(N \backslash B)$ extends $F \mid S^{2}$. The upshot is that $N$ can be recovered from an appropriate extension of $S^{2}$ in $\mathbf{R}^{3}$.

The outstanding problems concerning extensions revolve around determining relations between inequivalent extensions of a common immersion. For concreteness, we formulate a specific

Problem. Let $f: S^{2} \rightarrow \mathbf{R}^{3}$ be an immersion. Let $N_{i}^{\prime}, i=1,2$, be connected closed 3-manifolds, and let $N_{i}$ denote $N_{i}^{\prime}$ with a small open ball deleted. Suppose that $F_{i}: N_{i} \rightarrow \mathbf{R}^{3}, i=1,2$, are two inequivalent extensions of $f$. When are $N_{1}$ and $N_{2}$ diffeomorphic? 


\section{ACKNOWLEDGMENTS}

The author would like to thank the referee for his comments, which improved this paper considerably. Professor Kirby's interest in this work and the ensuing discussions were quite beneficial. I also appreciated discussing immersions with Professor Hirsch.

\section{REFERENCES}

1. G. Francis, Extensions to the disk of properly nested plane immersions of the circle, Michigan Math. J. 17 (1970). MR 44:2209

2. M. Hirsch, Immersions of manifolds, Trans. Amer. Math. Soc. 3 (1959), 242-276. MR 22:9980

3. _ Differential topology, Springer-Verlag, New York, 1976. MR 56:6669

4. J. Milnor and J. Stasheff, Characteristic classes, Princeton Univ. Press, 1974. MR 55:13428

5. J. Milnor, Morse theory, Princeton Univ. Press, 1963. MR 29:634

6. C. Pappas, The full two dimensional homotopy group, $\rho_{2}(X)$, preprint.

7. _ Extensions of immersions into Euclidean spaces, Thesis, Univ. of California, Berkeley, 1994.

8. V. Poénaru, Extension des immersions en codimension one (d'après S. Blank), Séminaire Bourbaki, 1968, no. 342.

9. S. Smale, On gradient dynamical systems, Ann. of Math. 74 (1961). MR 24:A2973

10. (1959), 327-344. MR 21:3862

11. J. H. C. Whitehead, The immersion of an open 3-manifold in Euclidean 3-space, Proc. London Math. Soc. (3) 11 (1961), 81-90. MR 23:A2224

Department of Mathematics, University of California, Berkeley, California 94720

E-mail address: pappas@math.berkeley.edu 